\title{
Diagnosing Lynch syndrome: is the answer in the mouth?
}

\section{H K Roy, H T Lynch}

\section{Alterations in vascular patterns in the buccal/subgingival mucosa may provide the basis for a non-invasive inexpensive test for recognising hereditary non-polyposis colorectal cancer}

$\mathrm{R}$ sk stratification is essential for designing efficacious and cost effective colon cancer screening programmes. One of the most important risk factors for colorectal cancers (CRC) is an inherited predisposition, implicated in $20 \%$ of all cases. ${ }^{1}$ The spectrum of genetic susceptibility ranges from the low penetrance mutations that modestly increase the colon cancer risk (for example, I $1307 \mathrm{~K})$ to the much more dramatic phenotypes (for example, multiple colonic adenomas in familial adenomatous polyposis) that engender an extraordinarily high risk of cancer. ${ }^{2}$ Lynch syndrome or hereditary non-polyposis colorectal cancer (HNPCC) is a case in point. This autosomal dominant condition results from a germline mutation in a DNA mismatch repair (MMR) gene (most commonly $h M L H 1, h M S H 2$, and $h M S H 6$ with the rare occurrence in $h M L H 3, P M S 1$, and PMS2). ${ }^{3}$ Clinically, Lynch syndrome, the most common hereditary disorder predisposing to CRC, is characterised by a greater than $80 \%$ lifetime risk of CRC in concert with an excess of several extracolonic cancers namely, endometrial, gastric, pancreatic, small bowel, ovarian, and transitional cell carcinoma of the upper uroepithelial tract (ureter and renal pelvis). Thus diagnosing Lynch syndrome is of considerable importance in order to institute a wide range of cancer surveillance strategies for affected subjects.

However, establishing the diagnosis is challenging and requires both considerable knowledge and vigilance. The potential reasons for overlooking the diagnosis of HNPCC include:

(1) Difficulty in many busy clinical practices of obtaining extended pedigrees necessary for identifying Lynch syndrome.

(2) Inadequate physician awareness of inherited colon cancer syndromes. ${ }^{4}$

(3) The variable phenotypic expression which may be modulated by both genetic and environmental factors. For instance, we have noted that subjects with hMLHl mutations have a higher risk of colon cancer than those with hMSH2 mutations (hazard ratio 2.0), and smokers who harbour germline MMR gene mutations are at an increased risk of colon cancer compared with non-smokers (hazard ratio 1.4) (Watson P, unpublished data).

(4) Suboptimal sensitivity and frequent ambiguity from germline testing (for example, approximately one third of hMLHl gene mutations are "missense mutations of unknown significance" $).^{5}$

(5) Limitations in the clinical criteria for diagnosing Lynch syndrome. For instance, Amsterdam II criteria (at least three members with HNPCC related cancer one of whom is a first degree relative of another affected, two successive generations, and one patient diagnosed before the age of 50 years) had a sensitivity of $78 \%$ and a specificity of $61 \%$ in the detection of established Lynch syndrome families. ${ }^{6}$ Although using less stringent criteria (for example, Bethesda) improved sensitivity, this came at the expense of specificity, thus potentially subjecting more families to germline testing. ${ }^{6}$

(6) Occurrence of new gene mutations, which would therefore lack a family history of CRC. For example, $11 \%$ of hMSH2 mutations are believed to be de novo events.

Thus because of these and other factors, Lynch syndrome is underdiagnosed and the relative paucity of identified HNPCC patients may not be reflective of the true incidence of the syndrome. The magnitude of this difference is not clear, but prevalence estimates suggest that approximately 1:350 to $1: 1700$ of the population are affected (calculated based on the assumptions that $1-5 \%$ of all CRCs are HNPCC related and the lifetime risk for an individual developing CRC is 6\%). This remarkably high population frequency estimate for Lynch syndrome is supported by a recent report that at least $1.6 \%$ of all endometrial cancers were related to germline mutations in
hMSH6. ${ }^{8}$ Germline hMSH6 mutations are responsible for approximately $10 \%$ of Lynch syndrome cases, ${ }^{9}$ suggesting that the total HNPCC related endometrial cancers may be considerably higher. Taken together, these lines of evidence indicate that Lynch syndrome is markedly under appreciated.

The clinical implications of this under recognition of Lynch syndrome can be devastating because of the high probability of developing malignancies in which the fatalities are potentially preventable. For instance, colonoscopic screening of HNPCC patients more than halved the risk of colon cancer, prevented all colon cancer deaths, and decreased overall mortality by $65 \% .^{10}$ We therefore recommend initiating colon cancer screening at age 25 years utilising colonoscopy because of the right sided predominance of colon lesions, repeating this annually because of the rapid adenoma to carcinoma transformation (accelerated carcinogenesis) that characterises Lynch syndrome. ${ }^{11}$ In women, we perform yearly transvaginal ultrasounds, endometrial aspirations, and CA 125 levels starting at age 30 years. Other screening recommendations are tailored to the specific issues related to family.

One useful tool that is becoming increasing employed for the detection of Lynch related colon cancers is microsatellite instability (MSI) analysis. MSIhigh status serves as the genetic fingerprint for DNA MMR defect, the hallmark of HNPCC related cancers. These studies can be expensive, are not universally available, and require access to tissue blocks. MMR insufficiency is extremely common in colon neoplasms. For instance, a survey of 209 CRCs demonstrated that $14 \%$ were MSI-high and $21 \%$ were MSI-low. ${ }^{12}$ Moreover, in patients with a family history of colon neoplasia (one first degree relative with colonic adenoma or CRC), 30\% of adenomatous polyps manifested high levels of MSI. ${ }^{13}$ Thus the positive predictive value of MSI analysis is low, subjecting many families to unnecessary germline mutational analyses, with the inherent expense and possible social and psychological ramifications. Indeed, an analysis was unable to formulate a strategy employing MSI and genetic testing for the diagnosis of HNPCC that would have both acceptable efficacy and cost effectiveness. ${ }^{14}$ Thus clearly other approaches are necessary to diagnose this condition.

The ideal marker for HNPCC would be sensitive, specific, non-invasive, and inexpensive. Many hereditary CRC predisposing conditions harbour easily recognisable physical findings that are useful for diagnosis. For instance, in 
Peutz-Jeghers syndrome, which has an approximate $10-38 \%$ lifetime incidence of CRC, the pigmented oral lesions are almost pathognomic. ${ }^{2}$ In familial adenomatous polyposis, where the lifetime CRC risk approaches $100 \%$, congenital hypertrophy of the retinal pigmented epithelium (CHRPE) has been an important clinical feature. ${ }^{15}$ Interestingly, while its presence connotes mutations in the centre of the $A P C$ gene (from exon 9 to 15), CHRPE has also been detected in familial polyposis unrelated to $A P C$ but associated instead with $M Y H$ mutations. ${ }^{16}$ Thus molecular pathogenesis responsible for the physical manifestations remains poorly understood. In HNPCC, there are no known easily detectable manifestations of physical examination (except for the sebaceous gland tumours in the rare HNPCC variant, Muir-Torre syndrome). ${ }^{17}$

In this issue of Gut, De Felice and colleagues $^{18}$ evaluate alterations in vascular patterns in the buccal/subgingival mucosa as a marker for HNPCC [see page 1764]. They reasoned that as blood vessel complexity increases in colon carcinogenesis, and Lynch syndrome represents a germline mutation and thus should be detectable ubiquitously, assessment of vascular complexity in remote areas of the body may be a screening tool for Lynch syndrome. High resolution pictures of buccal/subgingival mucosa from 14 patients from a Lynch II kindred and 30 healthy controls were obtained. These images of the vasculature were digitalised, and blood vessel complexity was determined. Using this methodology, they were able to demonstrate that there were highly statistically significant differences in patients from the Lynch II kindred compared with controls.

While obtaining the images is relatively straightforward, quantitating vascular complexity and geometry represents a much more formidable challenge. Previous studies utilising conventional Euclidean geometry (which relies on smooth shapes) were disappointing, largely because of the inability to approximate the irregularities inherent in malignancies. Fractal geometry, on the other hand, is far better suited in describing the somewhat random nature of tumour associated structures. ${ }^{19}$ Indeed, pathologists and cancer biologists are realising that accurate geometrical analysis of tumours, cells, and microvasculature can yield important information in the diagnosis and prognosis of malignancies. ${ }^{20}$ Fractal dimension is a well established measure of complexity and space filling nature of an object. The most widely used methodology for determining fractal dimension is box counting.
Boxes of one size are applied to the digitalised outline of an object and the number of squares required to cover objects are compared with that obtained with a different box size. ${ }^{19}$ With smooth objects, decreased box size corresponds closely with the increased box number which are required to cover the outline (for example, if the box size is half as much, then one needs $2^{2}$ or 4 times as many boxes). However, this relationship loses the proportionality in irregular objects. For example, with an irregular object such as a tumour, decreasing box size by half may only increase the needed box number by approximately threefold $\left(2^{1.6}\right)$. The exponent is a fraction instead of an integer and suggests that the object has fractal properties. Several groups demonstrated that fractal dimension of tumour vasculature is increased. ${ }^{20}$

Another measure of complexity used by De Felice and colleagues ${ }^{18}$ was chaos scores, which were also increased in Lynch syndrome patients. Chaos is somewhat of a misnomer and is better defined as "a form of order disguised as disorder". ${ }^{21}$ While chaotic systems are governed by simple rules of interactions, they are extremely sensitive to initial conditions and the slightest differences are magnified vastly at final outcomes. ${ }^{21}$ With regards to vasculature, the increase in fractal dimension and chaos scores in subjects with Lynch syndrome may be a marker of disorganised and tortuous microvessels, which have been previously reported in the vasculature supplying tumours. ${ }^{20}$

Are these mathematical parameters of any real clinical/biological relevance? Several groups have demonstrated that fractal analysis of histological slides can distinguish normal from malignant colonic tissue. ${ }^{22}$ It has been suggested that alterations in fractal dimension may be one of the earliest events in malignancy. ${ }^{23}$ Our preliminary report in experimental models of colon carcinogenesis strongly supports this claim. ${ }^{24}$ Others have postulated that there may be therapeutic implications for such parameters. For instance, optimisation of chemotherapy and radiation therapy require understanding the inefficiencies in tissue oxygenation and drug distribution related to the chaotic nature of tumour vascular architecture. ${ }^{20}$

Are these remarkable findings on the buccal vasculature complexity biologically plausible? This is difficult to definitively answer. While MSI-high tumours have distinctive pathological features, these do not encompass the blood vessel architecture. ${ }^{25}$ Indeed, one study suggested that Lynch syndrome tumours had less developed vasculature than sporadic colon cancers. ${ }^{26}$ To our knowledge, there have been no previous studies on blood vessel alterations in non-neoplastic areas. However, given the germline nature of the mutations, all endothelial cells should be affected. It is becoming increasingly clear that $h M L H 1$ and $h M S H 2$ have a wide number of other biological functions aside from DNA MMR. For example, DNA MMR enzymes have been implicated in the cellular apoptotic machinery. ${ }^{27}$ Endothelial apoptosis is an important process in governing cancer associated neovascularisation. ${ }^{28}$ While there are no reports on alterations in microvasculature in non-neoplastic epithelium, we have recently reported that increased mucosal blood flow may be a very early event in experimental colon carcinogenesis. ${ }^{24}$ Extrapolation from the uninvolved premalignant colonocytes to the buccal mucosal vasculature is somewhat tenuous. On the other hand, there are a variety of well established extraintestinal markers for hereditary colon cancer syndromes, including CHRPE for familial adenomatous polyposis, that do not have clear biological rationale.

There are several caveats in applying these remarkable data from De Felice and colleagues ${ }^{18}$ to clinical practice. One problem is that the Lynch II patients in this study were all related. Thus it is conceivable that the increased vascular complexity may be related to an inheritable trait unrelated to the presence of a DNA MMR gene mutation. This is suggested by the observation that members of these kindreds, believed not to have Lynch syndrome as ascertained by linkage analysis, also manifested the increased vascular complexity compared with controls. Indeed, in most parameters there were no significant differences between family members with and without mutations. However, the numbers were small. An alternative explanation of these inconsistencies may be that some of the "unaffected individuals" may potentially harbour mutations that were not detected by linkage analysis, a methodology with suboptimal sensitivity.

In summary, this provocative report leads to hope about the development of a non-invasive inexpensive test for recognising HNPCC. This would be of major importance in detecting heretofore unidentified patients and therefore initiating the intensive potentially life saving surveillance regimen. However, replication of these data in a large number of Lynch families is mandatory in order to translate this unique observation into clinical practice.

Gut 2003;52:1665-1667 


\section{Authors' affiliations}

H K Roy, Section of Gastroenterology, Evanston-Northwestern Healthcare Research Institute, Feinberg School of Medicine at Northwestern University, Evanston IL 60035, USA

H T Lynch, Department of Preventive Medicine and Public Health, Creighton University School of Medicine, Omaha, Nebraska, USA

Correspondence to: Professor H T Lynch, Department of Preventive Medicine and Public Health, Creighton University School of Medicine, Omaha, Nebraska, USA; htlynch@creighton.edu

\section{REFERENCES}

1 Lynch HT, de la Chapelle A. Hereditary colorectal cancer. N Engl J Med 2003;348:919-32.

2 Grady WM. Genetic testing for high-risk colon cancer patients. Gastroenterology 2003; 124:1574-94

3 Chung DC, Rustgi AK. The hereditary nonpolyposis colorectal cancer syndrome: genetics and clinical implications. Ann Intern Med 2003; 138:560-70.

4 Schroy PC 3rd, Barrison AF, Ling BS, et al. amily history and colorectal cancer screening: a survey of physician knowledge and practice patterns. Am J Gastroenterol 2002;97:1031-6.

5 Peltomaki P, Vasen HF. Mutations predisposing to hereditary nonpolyposis colorectal cancer: database and results of a collaborative study. The International Collaborative Group on Hereditary Nonpolyposis Colorectal Cancer.

Gastroenterology 1997;113:1146-58.

6 Syngal S, Fox EA, Eng C, et al. Sensitivity and specificity of clinical criteria for hereditary nonpolyposis colorectal cancer associated mutations in $\mathrm{MSH} 2$ and $\mathrm{MLH1}$. J Med Genet 2000;37:641-5.
7 Desai DC, Lockman JC, Chadwick RB, et al. Recurrent germline mutation in MSH2 arises frequently de novo. J Med Genet 2000; 37:646-52

8 Goodfellow PJ, Buttin BM, Herzog TJ, et al. Prevalence of defective DNA mismatch repair and MSH6 mutation in an unselected series of endometrial cancers. Proc Natl Acad Sci U S A 2003;100:5908-13.

9 Wagner A, Barrows A, Wijnen JT, et al. Molecular analysis of hereditary nonpolyposis colorectal cancer in the United States: high mutation detection rate among clinically selected families and characterization of an American founder genomic deletion of the $\mathrm{MSH} 2$ gene. Am J Hum Genet 2003:72:1088-100.

10 Jarvinen HJ, Aarnio M. Surveillance on mutation carriers of DNA mismatch repair genes. Ann Chir Gynaecol 2000;89:207-10.

11 Rijcken FE, Hollema H, Kleibeuker JH. Proximal adenomas in hereditary non-polyposis colorectal cancer are prone to rapid malignant transformation. Gut 2002:50:382-6.

12 Goel A, Arnold CN, Niedzwiecki D, et al. Characterization of sporadic colon cancer by patterns of genomic instability. Cancer Res 2003:63:1608-14.

13 Ricciardiello L, Goel A, Mantovani V, et al. Frequent loss of hMLH1 by promoter hypermethylation leads to microsatellite instability in adenomatous polyps of patients with a single first-degree member affected by colon cancer. Cancer Res 2003;63:787-92.

14 Reyes CM, Allen BA, Terdiman JP, et al. Comparison of selection strategies for genetic testing of patients with hereditary nonpolyposis colorectal carcinoma: effectiveness and costeffectiveness. Cancer 2002;95:1848-56.

15 King JE, Dozois RR, Lindor NM et al. Care of patients and their families with familial adenomatous polyposis. Mayo Clin Proc 2000;75:57-67.

16 Sieber OM, Lipton L, Crabtree M, et al. Multiple colorectal adenomas, classic adenomatous polyposis, and germ-line mutations in MYH. N Engl J Med 2003;348:791-9.
17 Fusaro RM, Lemon SJ, Lynch HT. Muir-Torre syndrome and defective DNA mismatch repair genes. J Am Acad Dermatol 1996;35:493-4.

18 De Felice C, Latini G, Bianciardi G, et al. Abnormal vascular network complexity: a new phenotypic marker in hereditary non-polyposis colorectal cancer syndrome. Gut 2003;52:000-0.

19 Cross SS. Fractals in pathology. J Pathol 1997; 182:1-8.

20 Baish JW, Jain RK. Fractals and cancer. Cancer Res 2000;60:3683-8.

21 Coffey DS. Self-organization, complexity and chaos: the new biology for medicine. Nat Med 1998:4:882-5.

22 Esgiar AN, Naguib RN, Sharif BS, et al. Fractal analysis in the detection of colonic cancer images. IEEE Trans Inf Technol Biomed 2002:6:54-8.

23 Waliszewski P, Molski M, Konarski J. On the holistic approach in cellular and cancer biology: nonlinearity, complexity, and quasi-determinism of the dynamic cellular network. J Surg Oncol 1998:68:70-8.

$24 \mathrm{Kim}$ YL, Liu Y, Wali RK, et al. Simultaneous measurement of angular and spectral properties of light scattering for the charterization of tissue microarchitecture and its alterations in early precancer. IEEE J Select Topics Quant Elec 2003 (in press)

25 Smyrk TC, Watson P, Kaul K, et al. Tumor infiltrating lymphocytes are a marker for microsatellite instability in colorectal carcinoma. Cancer 2001 :91:2417-22.

26 Losi L, Fante R, Di Gregorio C, et al. Biologic characterization of hereditary non-polyposis colorectal cancer. Nuclear ploidy, AgNOR count, microvessel distribution, oncogene expression, and grade-related parameters. Am J Clin Pathol 1995; 103:265-70.

27 Shimodaira H, Yoshioka-Yamashita A Kolodner RD, et al. Interaction of mismatch repair protein PMS2 and the p53-related transcription factor p73 in apoptosis response to cisplatin. Proc Natl Acad Sci U S A 2003;100:2420-5

28 Garcia-Barros M, Paris F, Cordon-Cardo C, et al Tumor response to radiotherapy regulated by endothelial cell apoptosis. Science 2003;300: 1155-9. 